\title{
On a Mapping Property of Some Singular Integral Operators in Sobolev-Slobodecky Spaces
}

\section{A. SEIF MSHIMBA}

The mapping properties $T_{D}: W_{m, p}(D) \rightarrow W_{m+1, p}(D)$ and $\Pi_{D}: W_{m, p}(D) \rightarrow W_{m, p}(D)(1<p<$ $+\infty ; m=0,1,2, \ldots)$ of the singular integral operators

$$
T_{D} f(z)=-\frac{1}{\pi} \iint_{D} \frac{f(\zeta)}{\zeta-z} d \xi d \eta \text { and } \Pi_{D} f(z)=-\frac{1}{\pi} \iint_{D} \frac{f(\zeta)}{(\zeta-z)^{-2}} d \xi d \eta \quad(z=x+i y, \zeta=\xi+i \eta)
$$

are generalized to arbitrary positive real values of $m$.

Key words: Singular integral operators, Sobolev spaces, Slobodecky spaces

AMS subject classification: $46 \mathrm{~B}, 46 \mathrm{E} .46 \mathrm{~F}$

The singular integral operators $T_{D}$ and $\Pi_{D}$ have been studied extensively in the spaces of Hölder-continuous functions $C_{m, \alpha}(\bar{D})$ and in the spaces of $p$-summable functions $W_{m, p}(D)$ $(m=0,1,2, \ldots$ and $1<p<+\infty ;$ see $[4,512-14])$. Under cetain assumptions on the domain $D, T_{D}$ and $\Pi_{D}$ map a function $f \in C_{m, \alpha}(\bar{D})$ into the spaces $C_{m+1, \alpha}(\bar{D})$ and $C_{m, \alpha}(\bar{D})$, respectively (see [13]). Recently the author has shown that $T_{D} g \in W_{m+1, p}(D)$ and $\Pi_{D} g \in W_{m, p}(D)$ if $g$ $\in W_{m, \rho}^{\prime}(D)$ and $D$ is a domain of finite area belonging to the class $C_{m}$ (see [7]). In this paper we establish that this property is maintained when restricted to Sobolev-Slobodecky spaces.

\section{The Sobolev-Slobodecky spaces $W_{a, p}(D)$}

The Sobolev-Slobodecky spaces, or more simply the Slobodecky spaces are a generalization of the more familar Sobolev spaces $W_{m, p}(D)$ where, while $p$ is still a real number in $(1,+\infty)$, the integer $m$ is now allowed to take any non-negative real value. They are thus commonly denoted by $W_{s, p}(D)(1 \leq p s+\infty ; s=[s]+d, 0 \leq d<1$ and [s] denoting, as usual, the largest integer not greater than $s)$. When $d=0$ the Sobolev-Slobodecky space $W_{s, p}(D)$ coincides with the Sobolev space $W_{s, p}(D)$. These spaces therefore fill the gaps between the spaces $L_{p}(D)$, $W_{1, \rho}(D), W_{2, p}(D), \ldots$ in essentially the same manner as the spaces of Hölder-continuous functions $C_{m, \alpha}(\bar{D})$ fill the gaps between the spaces of uniformly continuous functions $C_{m}(\bar{D})$. The Slobodecky spaces were introduced by L.N. Slobodecky, N. Aronsjan and E. Gagliardo in the $1950^{\circ}$ s and they play an important role in describing traces on planes and hyperplanes in $R^{n}$ of functions belonging to Sobolev spaces (see $[1,3,5,6,10,11]$ ).

A function $f$ defined in a domain $D \subset \mathbb{R}^{n}$ is said to belong to a Slobodecky space $W_{s, p}(D)$ $(1 \leq p<+\infty ; s>0)$ if
(i) $f \in W_{m, p}(D), m=[s]$
(ii) $\iint_{D D} \frac{\left|D^{\alpha_{f}}(x)-D^{\alpha_{f}}(y)\right|^{p}}{|x-y|^{n+(s-m) p}} d x d y<+\infty \quad\left(\alpha=\left(\alpha_{1}, \ldots, \alpha_{n}\right),|\alpha| \leq m\right)$. 
Equipped with the functional

$$
\|f\|_{s, p, D}=\|f\|_{m, p, D}+\sum_{|\alpha| \leq m}\left(\int_{D} \int_{D} \frac{\left|D^{\alpha} f(x)-D^{\alpha} f(y)\right|^{p}}{|x-y|^{n+(s-m)_{p}}} d x d y\right)^{1 / p}
$$

the set $W_{s, p}(D)$ becomes a Banach space (see $\left.[1,3,10,11]\right)$.

Similarly a function $f$ defined almost everywhere in a set $D \subset \mathbf{R}^{n}$ is said to belong to the set $W_{s, \infty}(D)(s \geq 0)$ if

$$
\text { (i) } f \in W_{m, \infty}(D), m=[s] \quad \text { (ii) ess sup } \frac{\left|D^{\alpha} f(x)-D^{\alpha} f(y)\right|}{|x-y|^{s-m}}<+\infty \forall x, y \in D, x \neq y,|\alpha| \leq m \text {. }
$$

In this case the norm may be defined as

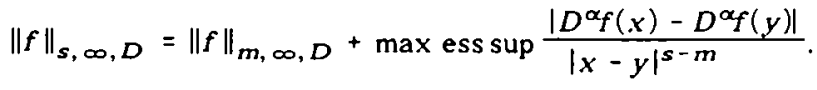

There are of course other equivalent definitions and norms for the Slobodecky spaces (see $[1,3,10,11])$.

Examplo: Every function $f \in C_{m, \beta}(\bar{D})$ belongs to the Slobodecky space $W_{s, p}(D)$ if $[s] \leq m$, $s-[s]<\beta$, and $p \geq 1$. This follows directly from the definitions of the two function spaces.

\section{Preliminaries}

For our investigations we shall need the following tools.

Lemma 1 (Schmidt's estimate): If $D$ is a planar domain of finite area $\mathrm{m} D$, then

$$
\iint_{D}|\zeta-z|^{-s} d \zeta d \eta \leq \frac{2 \pi}{2-s}\left(\frac{m D}{\pi}\right)^{1-s / 2} \text { for } 0 \leq s<2 \text { and any } z \in \bar{D} \text {. }
$$

A proof of the lemma involves the estimation of the given integral against the integral of the integrand over the disk of radius $R, R^{2}=\mathrm{m} D / \pi$, which is centred at the point $z$ (see $[5,9,12]$ for instance).

Lemma 2: If $D$ is a bounded domain in the plane, then for any two points $z_{j}, z_{k} \in \mathbb{C}$ and $\alpha, \beta$ $\epsilon[1,2)$ the following estimates hold:

$$
\iint_{D}\left|\zeta-z_{j}\right|^{-\alpha}\left|\zeta-z_{k}\right|^{-\beta} d \zeta d \eta \leq \begin{cases}M_{1}(\alpha, \beta, D)\left|z_{j}-z_{k}\right|^{2-\alpha-\beta} & \text { if } \alpha+\beta>2 \\ M_{2}(D)+M_{3}|\ln | z_{j}-z_{k}|| & \text { if } \alpha+\beta=2 \\ M_{4}(\alpha, \beta)(\operatorname{diam} D)^{2-\alpha-\beta} & \text { if } \alpha+\beta<2\end{cases}
$$

Proof: See $[5,12,13]$

Lemma 3: Let $h(x)=x^{\alpha} \ln x(x>0)$ and $\alpha=p(l-s)-r>0$ with $r \geq 0, p \geq 0$ and $0<s<1$. Then

a) $h$ has a relative minimum at $x_{0}=\cdot \exp (-1 / \alpha)$ and its value is $(-\alpha e)^{-1}$.

b) $h(x) \rightarrow-0$ as $x \rightarrow 0$.

Proof: These results can be derived directly using basic calculus 


\section{The mapping property}

First we consider the weakly singular integral operator $T_{D}$ in the Slobodecky space $W_{s, p}(D)$ $(0<s<1 ; 1 \leq p \leq+\infty)$. Thereafter a generalization to the case of arbitrary $s \geq 0$ will follow.

Let $D$ be a bounded domain in the complex plane. For $f \in L_{p}(D)$ we define

$$
T_{D} f(z)=-\frac{1}{\pi} \iint_{D} \frac{f(\zeta)}{\zeta-z} d \xi d \eta \quad(z=x+i y, \zeta=\xi+i \eta) .
$$

Case 1: $f \in L_{1}(D)$. It follows immediately from Fubini's theorem and the Schmidt estimate (Lemma 1) that

$$
\iint_{D}\left|T_{D} f(z)\right| d x d y \leq \frac{1}{\pi} \iint_{D}|f(\zeta)| d \xi d \eta \iint_{D}|\zeta-z|^{-1} d x d y \leq 2 \sqrt{\mathrm{m} D / \pi}\|f\|_{1, D} .
$$

Moreover, a repeated application of Fubini's theorem, Lemma 1 and Lemma 2 reveals that, for $\tau=t_{1}+t_{2}$,

$$
\begin{aligned}
& \iint_{D_{\tau}} \iint_{D_{z}} \frac{\left|T_{D} f(\zeta)-T_{D} f(z)\right|}{|\zeta-z|^{2+s}} d x d y d t_{1} d t_{2} \\
& \left.\quad \leq \frac{1}{\pi} \iint_{D_{\tau}}|f(\tau)| d t_{1} d t_{2} \iint_{D_{\zeta}}|\tau-\zeta|^{-1} d \xi d \eta \iint_{D_{z}} \mid \tau-z\right)\left.(\zeta-z)^{s+1}\right|^{-1} d x d y \\
& \quad \leq M(s, D)\|f\|_{1}, D \iint_{D_{\zeta}}|\tau-\zeta|^{-1-s} d \xi d \eta \\
& \quad \leq M(s, D) \frac{2 \pi}{1-s}\left(\frac{m D}{\pi}\right)^{(1-s) / 2}\|f\|_{1, D} .
\end{aligned}
$$

Case 2: $f \in L_{\infty}(D)$. It follows from Schmidt's estimate that $T_{D} f \in L_{\infty}(D)$ if $f \in L_{\infty}(D)$ and $D$ has a finite area. Indeed

$$
\left|T_{D} f(z)\right| \leq \frac{1}{\pi} \iint_{D}|f(\zeta)||\zeta-z|^{-1} d \xi d \eta \leq \underset{D}{\operatorname{ess} \sup |f|} \iint_{D}|\zeta-z|^{-1} d \xi d \eta \leq 2 \sqrt{\mathrm{m} D / \pi}\|f\|_{\infty}, D
$$

almost everywhere on $D$. Furthermore, by Lemma 2 and Lemma 3

$$
\begin{aligned}
\frac{\left|T_{D} f(z)-T_{D} f(\tau)\right|}{|z-\tau|^{s}} & \leq \frac{1}{\pi}|z-\tau|^{-1-s} \iint_{D} \frac{|f(\zeta)|}{|\zeta-z||\zeta-\tau|} d \xi d \eta \\
& \leq\left(M_{2}(D)+M_{3}|\ln | z-\tau||\right)\|f\|_{\infty, D}|z-\tau|^{1-s}<\infty \text { for all } z, \tau \in D .
\end{aligned}
$$

Before we move to show that $T_{D} f \in W_{s, p}(D), 1<p<+\infty$, if $f \in L_{p}(D)$, we note that, under certain assumptions on the boundary $\partial D$ of the domain $D, T_{D}$ maps $W_{m, p}(D)$ into $W_{m+1, p}(D), 1<$ $p<+\infty$ and $m=0,1,2, \ldots$ (see $[5,7]$ ).

Case 3: $f \in L_{p}(D), 1<p<2$. It follows from the Hölder inequality and Lemma 2 that

$$
\begin{aligned}
& \left|T_{D} f(z)-T_{D} f(\tau)\right| \\
& \quad \leq \frac{|z-\tau|}{\pi} \iint_{D}|f(\zeta)||(\zeta-z)(\zeta-\tau)|^{-1 / p}\left|(\zeta-z)(\zeta-\tau)^{-1 / q}\right| d \zeta d \eta
\end{aligned}
$$

12 Analysis, Bd. 11. Heft 2 (1992) 


$$
\begin{aligned}
& \leq \frac{|z-\tau|}{\pi}\left(\iint_{D}|f(\zeta)| P|(\zeta-z)(\zeta-\tau)|^{-1} d \xi d \eta\right)^{1 / P}\left(\iint_{D}|(\zeta-z)(\zeta-\tau)|^{-1} d \xi d \eta\right)^{1 / q} \\
& \leq\left(M_{2}(q)+M_{3}(q)|\ln | z-\tau||\right)^{1 / q}\left(\iint_{D}|f(\zeta)|^{P}|(\zeta-z)(\zeta-\tau)|^{-1} d \xi d \eta\right)^{1 / P}|z-\tau|
\end{aligned}
$$

Consequently we obtain

$$
\frac{\left|T_{D} f(z)-T_{D} f(\tau)\right|^{p}}{|z-\tau|^{2+s p}} \leq|z-\tau|^{p(1-s)-2}\left(M_{2}+M_{3}|\ln | z-\tau||\right)^{p / q} \iint_{D}|f(\zeta)|^{p}|(\zeta-z)(\zeta-\tau)|^{-1} d \xi d \eta,
$$

and thus by Lemma 3 and Lemma 1

$$
\begin{aligned}
& \iint_{D_{z}} \iint_{D_{\tau}}\left|T_{D} f(z)-T_{D} f(\tau)\right| P|z-\tau|^{-2-s p} d t_{1} d t_{2} d x d y \\
& \leq\left.\iint_{Z} \iint_{D_{\tau}} \iint_{D_{\zeta}}|f(\zeta)|^{p}|(\zeta-z)(\zeta-\tau)|^{-1}\left(M_{2}+M_{3}|\ln | z-\tau||\right)^{p^{\prime}} q_{z-\tau}\right|^{\alpha}|z-\tau|^{r^{-2}} d \xi d \eta d t_{1} d t_{2} d x d y \\
& \leq C_{1} \iint_{D_{\zeta}}|f(\zeta)|^{p} d \xi d \eta \iint_{D_{z}}|\zeta-z|^{-1} \iint_{D_{\tau}}|(\zeta-\tau)|^{-1}|z-\tau|^{r-2} d t_{1} d t_{2} d x d y \\
& s C_{1} M_{4}(r)\|f\|_{P}^{p} \iint_{D_{z}}|\zeta-z|^{r-2} d x d y \leq C(p, s, D)\|f\|_{P}^{P} \quad(r>0) .
\end{aligned}
$$

Case 4: $f \in L_{p}(D), 2<p<+\infty$. It follows from Hölder's inequality that

$$
\begin{aligned}
\left|T_{D} f(z)-T_{D} f(\tau)\right| & \leq \frac{|z-\tau|}{\pi} \iint_{D}|f(\zeta)||(\zeta-z)(\zeta-\tau)|^{-1} d \xi d \eta \\
& \leq \frac{|z-\tau|}{\pi}\|f\|_{p, D}\left(\iint_{D}|(\zeta-z)(\zeta-\tau)|^{-q} d \xi d \eta\right)^{1 / q} \\
& \leq M_{1}(p, D)\|f\|_{p, D}|z-\tau|^{(p-2) / p}
\end{aligned}
$$

(see $[5,12,13])$. Consequently

$$
\begin{aligned}
& \iiint_{D_{\tau}} \iint_{D_{z}}\left|T_{D} f(z)-T_{D} f(\tau)\right| P|z-\tau|^{-2-s p} d x d y d t_{1} d t_{2} \\
& \quad \leq\left(M_{1}(p, D)\|f\|_{p, D}\right)^{P} \iint_{D_{\tau}} \iint_{D_{z}}|z-\tau|^{P(1-s)-4} d x d y d t_{1} d t_{2}<+\infty,
\end{aligned}
$$

since the integral is weakly singular.

The results from the four cases above may be put together in the form of the following

Theorem 1: Suppose $f \in L_{p}(D), 1 \leq p \leq+\infty$. If $D$ is a bounded domain in the plane, then the weakly singular integral operator $T_{D}$ maps $f$ into the Slobodecky space $W_{s, p}(D), 0<s<1$, and

$$
\left\|T_{D} f\right\|_{s, p, D} \leq C(p, s, D)\|f\|_{p, D} .
$$

In view of the fact that $T_{D}$, under certain assumptions on the domain $D$, maps a function $f \in W_{m, p}(D)(m=0,1,2, \ldots, 1<p<+\infty)$ into the Sobolev space $W_{m+1, p}(D)$ (see $\left.[5-7]\right)$ we also have in our particular case $T_{D} f \in W_{1, p}(D)$. Since $f \in W_{s, p}(D) \subset L_{p}(D)$, it follows from the definition of the Slobodecky space that $T_{D} f \in W_{s+1, p}(D)$, and consequently $\Pi_{D} f \in W_{s, p}(D)$ $(0<s<1 ; 1<p<+\infty)$. 
Theorem 2: Suppose $f \in W_{s, p}(D)(0<s<1 ; 1<p<+\infty)$. If $D$ is a bounded domain in the plane, then the weakly singular integral operator $T_{D}$ maps $f$ into the Slobodecky space $W_{s+1, p}(D)$ and $\left\|T_{D} f\right\|_{s+1, p, D} \leq C(s, p, D)\|f\|_{s, p, D}$.

Theorem 3: The singular integral operator $\Pi_{D}$,

$$
\Pi_{D} f(z)=-\frac{1}{\pi} \iint_{D} \frac{f(\zeta)}{(\zeta-z)^{-2}} d \xi d \eta,
$$

maps the space $W_{s, p}(D)(0<s<1 ; 1<p<+\infty)$ into itself and $\left\|\Pi_{D} f\right\|_{s, p, D} s(s, p, D)\|f\|_{s, p, D}$.

We are now in position to generalize the theorems above for the case when $s \geq 1$.

Case 5: $f \in W_{s, p}(D)(s \geq 1 ; 2<p<+\infty)$. The function $T_{D} f$ is then Holder-continuous on $\bar{D} ; T_{D} f$ $\in C_{[s], \beta}(\bar{D}), \beta=(p-2) / p$ (see $\left.[5,13]\right)$. Moreover $T_{D} f \in W_{[s]+1, p}(D)$ (see [7]). As such, estimates similar to those under Case 4 hold, thus showing the validity of the generalization of Theorems 1 and 2 ; i.e. $T_{D} f \in W_{s+1, p}(D)$.

Case 6: $f \in W_{s, p}(D)(s \geq 1 ; 1<p \leq 2)$. Suppose [s] = 1. It then follows from Theorems 2 and 3 and the theorem from [7] cited above that

$$
T_{D} f \in W_{s+1, p}(D), \partial T_{D} f / \partial \bar{z}=f, \partial T_{D} f / \partial z=\Pi_{D} f \in W_{s, p}(D) .
$$

As such, estimates similar to those under Case 3 hold and we obtain $T_{D} f \in W_{s+1, p}(D)$.

By using mathematical induction we can conclude that $T_{D} f \in W_{s+1, p}(D)$ if $f \in W_{s, p}(D)$, for all $s \geq 1,1<p<+\infty$.

Since the integral operators $T_{D}$ and $\Pi_{D}$, under certain assumptions on the domain $D$, map a function $f \in W_{m, p}(D)\left(m \in \mathbb{N}_{0}, 1<p<+\infty\right)$ into the Sobolev spaces $W_{m+1, o}(D)$ and $W_{m, p}(D)$, respectively (see [5 - 7]), we can expand this statement as well as the Theorems 2 and 3 above to the

Theorem 4: Suppose $D \in C_{m}$ is a bounded domain in the plane. If $f \in W_{s, p}(D)(s \geq 0,[s]=$ $m ; 1<p<+\infty)$, then $T_{D} f \in W_{s+1, p}(D)$ and $\Pi_{D} f \in W_{s, p}(D)$ as well as

$$
\left\|T_{D} f\right\|_{s+1, p, D} \leq C(s, p, D)\|f\|_{s, p, D} \text { and }\left\|\Pi_{D} f\right\|_{s, p, D} \leq A(s, p, D)\|f\|_{s, p, D} \text {. }
$$

Suppose $f \in W_{s, p}(D)(1 / p<s<+\infty ; 1<p<+\infty)$. If the domain $D$ belongs to the class $C_{m}$, $m=[s]$, then it follows from the Ostrogradskii-Gauss theorem that

$$
\begin{aligned}
\Pi_{D} f(z) & =-\frac{1}{\pi} \iint_{D} f(\zeta)(\zeta-z)^{-2} d \xi d \eta=\lim _{\varepsilon \rightarrow 0}\left\{\iint_{D_{\varepsilon}} \frac{\partial}{\partial \zeta} \frac{f(\zeta)}{\zeta-z} d \xi d \eta-\iint_{D_{\varepsilon}} \frac{\partial f}{\partial \zeta} \frac{d \xi d \eta}{\zeta-z}\right\} \\
& =\lim _{\varepsilon \rightarrow 0}\left\{-\frac{1}{\pi} \iint_{D_{\varepsilon}} \frac{\partial f}{\partial \zeta} \frac{d \xi d \eta}{\zeta-z}+\frac{1}{2 \pi \mathrm{i}} \int_{\Gamma} \frac{f(\zeta)}{\zeta-z} d \bar{\zeta}-\frac{1}{2 \pi \mathrm{i}} \int_{\partial D} \frac{f(\zeta)}{\zeta-z} d \bar{\zeta}\right\} \\
& =T_{D}\left(\frac{\partial f}{\partial \zeta}\right)=\frac{1}{2 \pi \mathrm{i}} \int_{\partial D} \frac{f(\zeta)}{\zeta-z} d \zeta,
\end{aligned}
$$

where $D_{\varepsilon}=D \cap\{\zeta:|\zeta-z|>\varepsilon\}$ and $\Gamma=\{\zeta:|\zeta-z|=\varepsilon\}$. It thus follows from Theorem 4 that the Cauchy type integral $\frac{1}{2 \pi \mathrm{i}} \int_{\partial D} f(\zeta)(\zeta-z)^{-1} d \bar{\zeta}$ belongs to the Slobodecky space $W_{s, p}(D)(1 / p<s$ 
$<+\infty ; 1<p<+\infty)$. On the other hand, the traces of $f$ on the boundary $\partial D$ belong to the Slobodecky space $W_{s-1 / p, p}(\partial D)$ (see $\left.[10,11]\right)$. We are thus led to the following

Theorem 5: If $D \in C_{[s]}$ and $f \in W_{s-1 / p, p}(\partial D)(1 / p<s<+\infty ; 1<p<+\infty)$, then the Cauchy type integral

$$
\Phi(z)=\frac{1}{2 \pi \mathrm{i}} \int_{\partial D} \frac{f(\zeta)}{\zeta-z} d \zeta
$$

belongs to the function space $W_{s, p}(D)$.

Romark: Theorem 5 presents a generalization of analogous Theorems in $C_{m, \alpha}(\bar{D})$ and $W_{k, p}(D)$ for $m \in \mathbb{N}_{0}$ and $k \in \mathbb{N}$ (see $[7,13]$ ).

Acknowledgement: This work was carried out during the author's visit to the International Centre for Theoretical Physics in Trieste. Italy, in his capacity as Associate to the Centre. The author expresses his thanks to the Director of the Centre, Prof. Abdus Salam, for the suppert.

\section{REFERENCES}

[1] ADAMS, R.A.: Sobolev Spaces. New York: Academic Press 1975

[2] CALDERON, A. P.: Cauchy integrals on Lipschitz curves and related operators. Proc. Nat. Acad. Sci. USA 74 (1977), 1324 - 1327.

[3] KUFNER, A., JOHN, O. and S. FǓCIK: Function Spaces. Leyden: Noordhof Int. Publ. 1977.

[4] LANCKAU, E. and W. TUTSCHKE: Complex Analysis-Methods, Trends and Applicarions. Oxford: North Oxford Acade mic 1985.

[5] MSHIMB^. A.S.: Konstruktion von Lösungen nichtlinearer elliptischer Differentialgleichungssysteme erster Ordnung in der Ebene durch komplexe Methoden im Sobolev-Raum $W_{l, p}(G)$. Dissertation. Halle: Martin-Luther-Universität Halle-Wittenberg 1979.

[6] MSHIMBA, A.S.: Construction of the solution to the Dirichlet boundary value problem in $W_{l, p}(G)$ for systems of partial differential equations in the plane. Math. Nachr. 99 (1980), 146.163.

[7] MSHIMBA, A.S.: On a mapping property of the singular integral operators $T_{D}$ and $\Pi_{D}$ in Sobolev spaces. Math. Nachr. 147 (1990), 153 - 159.

[8] NERI, U.: Singular Integrals. Lect. Notes Math. 200 (1971), 1 - 272.

[9] SCHMIDT, E.: Bemerkungen zur Potentialtheorie. In: Matheatische Abhandlungen Hermann Amandus Schwarz. Festschrift zum 50. Geburtstag. Berlin: Verlag J. Springer 1914 , pp. $365-383$.

[10] TRIEBEL. H.: Interpolation Theory. Function Spaces, Differential Operators. Amsterdam: North Holland Publ. Comp. 1978.

[11] TRIEBEL, H.: Theory of Function Spaces. Basel: Birkhäuser Verlag 1983.

[12] TUTSCHKE. W.: Partielle Differentialgleichungen (Teubner - Texte zur Mathematik: Vol. 27). Leipzig: B. G. Teubner Verlagsges. 1983.

[13] VekUa, I.N.: Generalized Analytic Functions. Oxford: Pergamon Press 1962.

[14] ZYGMUND, A. and A.P. CALDERON: On the existence of singular integrals. Acta Math. 88 (1952), $85-139$.

Received 21.01.1991

Dr. Ali Seif Msimba

University of Dar es Salaam, Department of Mathematics

P. O. Box 35062

Dar es Salaam, The United Republic of Tanzania 